\title{
Prevalence, recovery and factors associated with smell and taste disruption in patients with coronavirus disease 2019
}

\author{
Pablo Parente-Arias ${ }^{1}$, Pilar Barreira-Fernandez ${ }^{1}$, Ana Quintana-Sanjuas ${ }^{1}$, and Berta \\ Patiño-Castiñeiras ${ }^{1}$ \\ ${ }^{1}$ Hospital Universitario Lucus Augusti
}

June 2, 2020

\begin{abstract}
Abstract Objective: To determine the occurrence of olfactory and gustatory disruptions in COVID-19 patients, their association with demographic and clinical features and prognosis. Design: This observational cohort study involved consecutively diagnosed COVID-19 patients tested between March the 3rd 2020 and March the 24th 2020. Setting: A geographically defined cohort area. All COVID-19 patients were evaluated in a University Hospital. Participants: From the first 184 consecutive patients, 32 were considered ineligible and one refused to participate. Main Outcomes and Measures: The primary outcome of interest is the prevalence of smell and taste alterations, factors associated and recovery rate. Univariate and multivariate analysis by logistic regression was performed to detect factors associated to these symptoms. Results: 151 patients were included and $99(65.3 \%)$ reported olfactory or gustatory symptoms. Olfactory dysfunction was reported by 75 patients (49.7\%). Isolated anosmia was found in 2 patients (1.3\%). Gustatory dysfunction was reported by 91 patients (60.3\%). Factors associated with higher prevalence of smell dysfunction included age, sex, and comorbidities. The time to smell disruption development was significantly shorter in mild to moderate patients than in severe patients $(\mathrm{p}=0.043)$. In $85.3 \%$ of patients with smell disruption, the symptom had been resolved in the first 2 months Conclusions and Relevance: Olfactory and gustatory dysfunction was common in COVID-19 patients. Smell disruption has high recovery rate and was associated with age, sex, and clinical severity. It may be beneficial to investigate the appearance of taste and/or smell disruptions in individual patients, with respect to diagnosis and prognosis.
\end{abstract}

\section{Key points}

- Prevalence of smell and taste alterations was $65,3 \%$.

- Smell alteration was associated with age, sex and comorbidities. Only sex was found to be associatedwith taste alteration.

- The time elapsed from disease onset to anosmia or hyposmia development was associated with clinical severity.

- Recovery rate after a 2 months follow-up was $85.3 \%$

Keywords : coronavirus; COVID-19; SARS-CoV-2; smell; taste; gustatory; olfactory.

\section{Introduction}

At the end of 2019, some patients in Wuhan, China developed bilateral pneumonia that was subsequently determined to be caused by a novel coronavirus, now named severe acute respiratory syndrome coronavirus 2 (SARS-CoV-2) ${ }^{1}$. The disease it causes has been named coronavirus disease 2019 (COVID-19), and it has become a pandemic that has rapidly spread virtually worldwide. The virus is transmitted via oronasal cavities $^{2}$ through aerosols and droplets from both symptomatic and asymptomatic patients ${ }^{3,4}$. The most 
common symptoms reported in China include fever, coughing, dyspnea, sputum production, myalgia, arthralgia, headache, and diarrhea. Otolaryngologic symptoms were less prevalent in those studies but included sore throat (7\%-15\%), nasal discharge (5\%), nasal obstruction (5\%), and anosmia and/or ageusia $(5 \%)^{5}$.

Smell and taste dysfunction have been reported by otolaryngologists in COVID-19 patients, sometimes as the sole symptoms present, and this has led to issue specific recommendations in this regard. Some evidence has emerged implicating both olfactory and gustatory disruptions as indicative clinical symptoms in COVID-19 patients outside China.

The aims of the current study were to investigate and characterize the occurrence, duration and recovery of olfactory and gustatory disruptions in a geographically defined cohort of patients with laboratory-confirmed COVID-19and investigate therelationships between these disruptions and demographic and clinical features.

\section{MATERIALS AND METHODS}

\section{Study Design}

Anobservational cohort study involving consecutively diagnosed COVID-19patientsbetween March the $3^{\text {rd }}$ 2020 and March the $24^{\text {th }} 2020$ in the province of Lugo(Spain) was performed.

\section{Participants}

Inclusion criteria were age $>18$ years and COVID-19 confirmed via reverse transcription polymerase chain reaction (RT-PCR). The exclusion criteria were inability to speak on the telephone, olfactory or gustatory dysfunction prior to the COVID-19 epidemic, and lack of informed consent. Insufficient cognitive skills to collaborate were also considered an exclusion criterion. Cognitive skills were assessed via interview (subjectively assessed by the evaluator) and from medical records.

\section{Measures}

Initial telephone interviews were conducted between April the $1^{\text {st }} 2020$ and April the $10^{\text {th }} 2020$ by four researchers. Form April the $14^{\text {th }} 2020$ to May $25^{\text {th }} 2020$ researchers re-contacted patients who had reported persistence of symptoms during the first telephone interview, to assess whether they had recovered or were experiencing ongoing persistence.

Electronic charts were reviewed during telephone interviews and all relevant clinical data were confirmed by the patient. The questionnaire included age, sex, date of clinical onset, date of testing, general clinical symptoms (fever, dry cough, dyspnea, diarrhea, myalgia, among others) and their dates of onset, presence of otolaryngologic symptoms (rhinorrhea, nasal obstruction, sore throat, otalgia, facial pain), and medical history (specific otolaryngologic history, comorbidities, previous chronic treatment, current treatment). Based on potentially relevant laboratory test results, imaging, and clinical data obtained from electronic records, patients were classified in accordance with Wang et al. ${ }^{6}$, and assigned to a mild group (mild to moderate in Wang's classification) or to a severe group (severe and critical in Wang's classification).

Questions pertaining to olfactory impairment were based on the Self-reported Mini Olfactory Questionnaire ${ }^{7}$, but were adapted to be applied to isolated patients. Ability to smell sanitary alcohol, bleach, perfume and coffee were assessed. Based on patients' answers they were assigned to a no symptoms group (no change in their ability to recognize usual smells), hyposmicgroup (who experienced changes in their sensitivity but that they still had maintained perception of strong odors), or anosmic group (unable to perceive any smells). Taste impairment was measured by means of relevant questions featured in the United States National Health and Nutrition Examination survey ${ }^{8}$. Based on the patients' answers they were assigned to a no symptoms group (no change in their ability to perceive taste), a dysgeusia group (alteration or distortion of their ability to taste), or an ageusia group (their ability to taste was reduced or abolished).

Statistical methods 
Statistical analyses were performed using version 3.5.2 of the $\mathrm{R}$ programming language (R Project for Statistical Computing; R Foundation).Relationships between variables were assessed via the chi-square test. The Kaplan-Meier method was used to generate curves to estimate the timing of the emergence of symptoms, and curves derived from patients with mild disease were compared with curves derived from patients with severe disease via the log-rank test. Lastly, the comparative influences of different factors on disease's severity were investigated using logistic regression, associated odd-ratios, and $95 \%$ confidence intervals.

\section{RESULTS}

The first 184 patients who tested positive in the first 1,007 PCR tests performed at Lugo were considered for inclusion in the study, but 33 were excluded based on the aforementioned criteria. Of these 33 patients, 9 had been intubated in the intensive care unit during the study, 7 were in the intensive care unit and their clinical circumstances rendered participation in the interview impossible, 7 had died, 4 were uncontactable, 3 had dementia, 2 were aged $<18$ years, and 1 refused to participate. A total of 151 patients completed the study, 53 men and 98 women, and their mean age was 55.2 years (range 18-88 years). Thirty-five patients were included inthesevere group because they fulfilled the requirements at some point during the evolution of their disease condition. Patient characteristics and comorbidities are summarized in Table 1, and non-mutually exclusive general and otolaryngologic symptoms are presented in Table 2.

Of the 151 patients who completed the study, 99 (65.6\%) reported olfactory or gustatory symptoms. Of these, $67 / 99(67.7 \%)$ reported loss of either smell or taste, 8/99 (8.1\%) reported just smell alterations and 24/99 (24.2\%) reported only taste alterations (Table 3 ).

Olfactory dysfunction was reported by $75 / 151$ patients (49.7\%), including $49(32.5 \%)$ who reported anosmia and $26(17.2 \%)$ who reported hyposmia. Isolated anosmia (neither general nor otolaryngological symptom unless anosmia) was found in 2 patients (1.3\%). Olfactory dysfunction was significantly associated age $(63.2 \%$ of patients aged $<60$ years vs. $31,2 \%$ aged [?] 60 years, $p=0.028)$, sex $(59.1 \%$ in women vs. $32.0 \%$ in men, $p=0.003$ ), and comorbidities (61.9\% in patients with no comorbidities; $39.4 \%$ with one, $\mathrm{p}=0.031 ; 24.0 \%$ with two, $\mathrm{p}=0.049)$, but not with clinical severity $(54.3 \%$ of mild to moderate patients vs. $34.2 \%$ of severe patients, $p=0.3$ ) (Table 3 ). Anosmia or hyposmia developed on the same day as the rest of the symptoms in 19/75 patients (25.3\%). In the rest of the patients time from clinical onset to olfactory disruption varied from 1 day to 11 days (mean $4.4 \pm 0.6$ days). The time to anosmia or hyposmia developmentwas significantly shorter in mild to moderate patients than in severe patients $(p<0.05$; Figure 1). Although all patients reported improvement, in $11 / 75$ (14.7\%) patients the symptom remained at the final follow-up timepoint, which constituted durations ranging from 61 to78 days (mean $70.5 \pm 3.3$ days). In those in whom the symptom had resolved by the final follow-up timepoint, it had lasted from 3 to 60 days (mean $17.7 \pm 8.9$ days), $55,2 \%$ of them in the first 12 days.Figure 2 .

Gustatory dysfunction was reported by $91 / 151$ patients (60.3\%), including 59 (39.1\%) who reported ageusia and $32(21.2 \%)$ who reported dysgeusia. The presence of ageusia or dysgeusia was only significantly associated with sex ( $68.3 \%$ in women vs. $43.4 \%$ in men, $p=0.028)$.

\section{DISCUSSION}

Synopsis of key findings

The present study adds evidence to the knowledge base pertaining to alterations in smell and tasteand COVID 19, and,to our knowledge, it's the first study to find association between these symptoms and age, sex, disease severity, and comorbidities.The rate of olfactory and/or gustatory dysfunction found was $65.6 \%$ ( $49.7 \%$ olfactory and $60.3 \%$ gustatory), showing a recovery rate of $85.3 \%$ after, at least, 61 days, in the longest follow up reported.

Comparisons with other studies

The prevalence of such symptoms varies markedly between studies, from $5.1 \%$ to $98.0 \%{ }^{9}$.Several factors are involved in this variation. The most influential is the technique used to assess the presence of olfactory or 
gustatory disruption. Chart review studies were initially used by Vaira et al. ${ }^{10}$ and Mao et al. ${ }^{11}$ and reported prevalenceof chemosensory dysfunction from $5,1 \%$ to $19.4 \%$. Records-based investigations underestimate such symptoms because assessment was not included in previous clinical protocols.Self-reporting of symptoms via online questionnaires has been used byLechien et al. ${ }^{12}$ reporting $85.6 \%$ olfactory dysfunction and $88.0 \%$ gustatory dysfunction. Notably, however, self-reported olfactory acuity is reportedly inaccurate ${ }^{13}$. Some studies have assessed symptoms via oral interviews. Giacomelli et al. ${ }^{14}$ interviewed 59 hospitalized patients, and $33.9 \%$ reported at least one taste or olfactory disorder and $18.6 \%$ reported both.Although they may both underestimate or overestimate olfactory acuity ${ }^{15}$, to study the alteration of smell as a symptom of COVID-19 it is crucial that the patient is aware of the disruption and can convey it during an anamnesis. Visual scales may yield more accurate measures of smell and taste than most other methods ${ }^{16}$. Yan et al. ${ }^{17}$ used an online visual scaleto compare olfactory and gustatory functions in COVID-19-positive and COVID-19-negative patients and reported olfactory loss in $68 \%$ and gustatory loss in $71 \%$ of COVID-19-positive patients and $16 \%$ and $17 \%$ in negative subjects. Validated quantitative olfactory testing is considered the most accurate method of assessment. Moein et al have used it to compare COVID-19-positive patients with COVID-19-negative and found that $98 \%$ of COVID-19-positive patientshad objective olfactory disruption although only $35 \%$ of the patients reported smell and or taste disruption previouly ${ }^{18}$

Different recruitment tools may yield different study results. Recruiting a sample that includes all infected patients (from asymptomatic to critical) is very difficult. Some studies are very weak in this respect because participants have been recruited via the internet, leaflets, and/or word-of-mouth strategies whereby the patient can become aware of the aims of the study ${ }^{12}$. Other studies have tried to overcome these limitations through different strategies pertaining to demographic and clinical characteristics of the sample ${ }^{12,17}$ and comparisons with other clinical studies ${ }^{18}$.

In the present study, there were associations between these disruptions and age, sex, and disease severity. Age is known to be associated with olfactory $\operatorname{loss}^{15}$. All patients in the present study reported normal olfactory and gustatory function before COVID-19 onset, and disruption was based on differential acuity thereafter. Only one previous study has shown this ${ }^{14}$. Some evidence suggests that olfactory capacity is worse in men than in women at all ages ${ }^{15}$. In the COVID-19 patients in the current study, olfactory and gustatory disruptions were more common in women than in men, as it has been reported in other studies ${ }^{12,14}$, but this was not found in objective testing studies ${ }^{18}$. It could mean that women can be aware of smell and taste alteration better than men.

In our study we found an association between presence of comorbidities and clinical severity and smell disruptions. Patients with one or more comorbidities reported less smell and taste disruptions andthe timing of presentation differed between severe and non-severe patients. Mild and moderate patients reported an earlier onset of olfactory dysfunction. This has not been reported in previous studies even if Yan et al ${ }^{19}$ reported that anosmia was strongly and independently associated with outpatient care. The meaning of the observation is not clear. One proposed pathophysiological mechanism of anosmia involves a bigger local inflammatory response the first days of the infection, mediated by upregulated expression of angiotensin-converting enzyme 2 (ACE2), which increases the risk of coronavirus infection, resulting in greater olfactory dysfunction but protecting against lung injury, as it was proposed in smokers ${ }^{20}$.

In the present study, smell and taste disruptions occurred at various points during disease progression. The existence of a common syndrome associated with COVID-19 infection termed isolated sudden onset anosmia has been proposed in some studies ${ }^{21}$. Notably, only 2 patients in the present study reported other nonolfactory and non-gustatory symptoms.Probably,exhaustiveinterview with simultaneous access to clinical electronic data and close follow up allowed to identify other symptoms associated. There is a lack of evidence about the prognosis of olfactive disruption in COVID-19. Hopkins et al ${ }^{22}$ stated that $80.1 \%$ of patients reported improvement in one week.

The pathophysiology of olfactory and gustatory disruption in COVID-19 patients remains unclear. In the olfactory system, "sustentacular cells"stem cells and perivascular cells, but not in olfactory sensory neurons or olfactory bulb neurons, constitute a prime candidate for SARS-CoV-2 infection via the nose, and possibly 
for SARS-CoV-2-induced anosmia, because,like respiratory ciliated cells, they both have ACE2 receptors and membrane-anchored serine protease 2 , which are uncommon characteristics in other human tissues. Via these components, nerve dysfunction may be mediated by damage to support cells and ensuing inflammation, without neuronal infection, or altering neurotransmission in the absence of neurosensory cell death ${ }^{23} \mathrm{enabling}$ regeneration, fluctuation and spontaneous improvement. Taste sensory receptors may be affected in a similar way, because ACE2 receptors are expressed in the oral mucosa ${ }^{24,25}$.

Strengths of the study

The main aim of the present study was to investigate a representative sample of patients that was minimally affected by the usual sources of recruitment bias. Thus,it included all patients who tested positive for COVID-19 in the first test performed during a short period in a specific geographical area and resulted in a representative sample with regard to age, sociocultural background, and disease severity. We consider telephone interviews based on a validated questionnaire the most reliable way to assess the presence of smell and taste alteration (not acuity), both in home-isolated patients and in hospitalized patients and allow a close follow up of the patients that adds information about duration and recovery.

Potential weakness of study

The sample was relatively small and geographically limited. The method used to assess olfactory and gustatory dysfunction is not the most accurate. Ideally, an electrophysiological method or sniffing test that can quantify smell and taste acuity and accurately distinguish between olfactory and gustatory symptoms would be used. Nevertheless, such methods require direct interaction between patients and researchers and are thus impractical in patients who are isolated at home or in hospital and may be unnecessary if dysfunction can be reliably established via other patient-based methods, irrespective of significance or effects on prognosis. Although it is the longest follow-up period published, whether the persistent olfactory or gustatory disruption reported by some patients at the last interview will be permanent remains to be determined.

Clinical applicability of the study

These results suggest that during the current pandemic it may be beneficial to investigate the presence and time of appearance of smell disruptions in individual patients, with respect to initial diagnosis, preventive isolation and clinical prognosis. Smell disruption has showed less value. For patients, these disruptions have good prognosis as they improved in all patients, with a complete recovery in $85,3 \%$ in less than two months.

\section{Conclusion}

Olfactory and gustatory dysfunction in COVID-19 patients has an high prevalence and smell disruption is associated withand age, sex, comorbidities and time to appearance is associated with clinical severity. Although all patients improved, some of them reports alteration after two months follow-upsofurther research is needed.

\section{References}

1. Zhu N, Zhang D, Wang W, et al. A Novel Coronavirus from Patients with Pneumonia in China, 2019. $N$ Engl J Med. 2020;382(8):727-733.

2. Wang W, Xu Y, Gao R, et al. Detection of SARS-CoV-2 in Different Types of Clinical Specimens. JAMA. 2020 .

3. Hu Z, Song C, Xu C, et al. Clinical characteristics of 24 asymptomatic infections with COVID-19 screened among close contacts in Nanjing, China. Sci China Life Sci. 2020.

4. Lauer SA, Grantz KH, Bi Q, et al. The Incubation Period of Coronavirus Disease 2019 (COVID-19) From Publicly Reported Confirmed Cases: Estimation and Application. Ann Intern Med. 2020.

5. Guan WJ, Ni ZY, Hu Y, et al. Clinical Characteristics of Coronavirus Disease 2019 in China. N Engl J Med. 2020. 
6. Wang Y, Liu Y, Liu L, Wang X, Luo N, Li L. Clinical Outcomes in 55 Patients With Severe Acute Respiratory Syndrome Coronavirus 2 Who Were Asymptomatic at Hospital Admission in Shenzhen, China. The Journal of Infectious Diseases. 2020.

7. Zou LQ, Linden L, Cuevas M, et al. Self-reported mini olfactory questionnaire (Self-MOQ): A simple and useful measurement for the screening of olfactory dysfunction. Laryngoscope. 2019.

8. Bhattacharyya N, Kepnes LJ. Contemporary assessment of the prevalence of smell and taste problems in adults. Laryngoscope.2015;125(5):1102-1106.

9. Tong JY, Wong A, Zhu D, Fastenberg JH, Tham T. The Prevalence of Olfactory and Gustatory Dysfunction in COVID-19 Patients: A Systematic Review and Meta-analysis. Otolaryngol Head Neck Surg.2020:194599820926473.

10. Vaira LA, Salzano G, Deiana G, De Riu G. Anosmia and Ageusia: Common Findings in COVID-19 Patients. Laryngoscope. 2020.

11. Mao L, Jin H, Wang M, et al. Neurologic Manifestations of Hospitalized Patients With Coronavirus Disease 2019 in Wuhan, China.JAMA Neurology. 2020.

12. Lechien JR, Chiesa-Estomba CM, De Siati DR, et al. Olfactory and gustatory dysfunctions as a clinical presentation of mild-to-moderate forms of the coronavirus disease (COVID-19): a multicenter European study. Eur Arch Otorhinolaryngol. 2020.

13. Temmel AFP, Quint C, Schickinger-Fischer B, Klimek L, Stoller E, Hummel T. Characteristics of Olfactory Disorders in Relation to Major Causes of Olfactory Loss. Archives of Otolaryngology-Head $\mathscr{E}$ Neck Surgery. 2002;128(6):635-641.

14. Giacomelli A, Pezzati L, Conti F, et al. Self-reported Olfactory and Taste Disorders in Patients With Severe Acute Respiratory Coronavirus 2 Infection: A Cross-sectional Study. Clinical Infectious Diseases.2020.

15. Bramerson A, Johansson L, Ek L, Nordin S, Bende M. Prevalence of olfactory dysfunction: the skovde population-based study.Laryngoscope. 2004;114(4):733-737.

16. Langdon C, Lehrer E, Berenguer J, et al. Olfactory Training in Post-Traumatic Smell Impairment: Mild Improvement in Threshold Performances: Results from a Randomized Controlled Trial. J Neurotrauma. 2018;35(22):2641-2652.

17. Yan CH, Faraji F, Prajapati DP, Boone CE, DeConde AS. Association of chemosensory dysfunction and Covid-19 in patients presenting with influenza-like symptoms. Int Forum Allergy Rhinol. 2020.

18. Moein ST, Hashemian SMR, Mansourafshar B, Khorram-Tousi A, Tabarsi P, Doty RL. Smell dysfunction: a biomarker for COVID-19. Int Forum Allergy Rhinol. 2020.

19. Yan CH, Faraji F, Prajapati DP, Ostrander BT, DeConde AS. Self-reported olfactory loss associates with outpatient clinical course in Covid-19. Int Forum Allergy Rhinol. 2020.

20. Leung JM, Yang CX, Tam A, et al. ACE-2 Expression in the Small Airway Epithelia of Smokers and COPD Patients: Implications for COVID-19. Eur Respir J. 2020.

21. Gane SB, Kelly C, Hopkins C. Isolated sudden onset anosmia in COVID-19 infection. A novel syndrome? Rhinology. 2020.

22. Hopkins C, Surda P, Whitehead E, Kumar BN. Early recovery following new onset anosmia during the COVID-19 pandemic - an observational cohort study. J Otolaryngol Head Neck Surg. 2020;49(1):26.

23. Finsterer J, Stollberger C. Causes of hypogeusia/hyposmia in SARS-CoV2 infected patients. J Med Virol. 2020. 
24. Xu H, Zhong L, Deng J, et al. High expression of ACE2 receptor of 2019-nCoV on the epithelial cells of oral mucosa. Int J Oral Sci.2020;12(1):8.

25. Vaira LA, Salzano G, Fois AG, Piombino P, De Riu G. Potential pathogenesis of ageusia and anosmia in COVID-19 patients. Int Forum Allergy Rhinol. 2020.

Figure 1: Kaplan Meyer appearance of smell disruption estimates based on mild and severe disease.

Figure 2: Days to complete recovery of smell and/or taste disruption

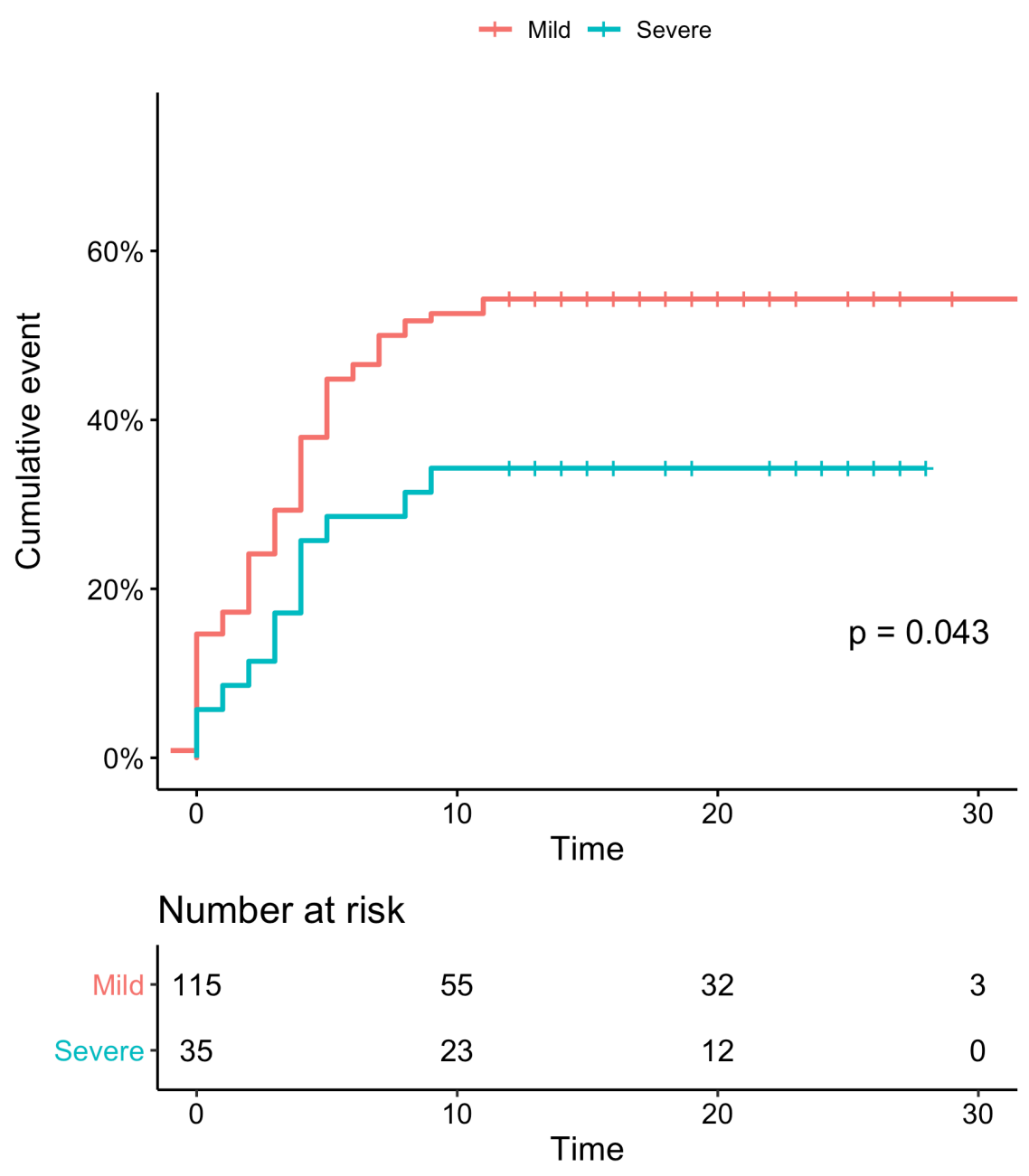




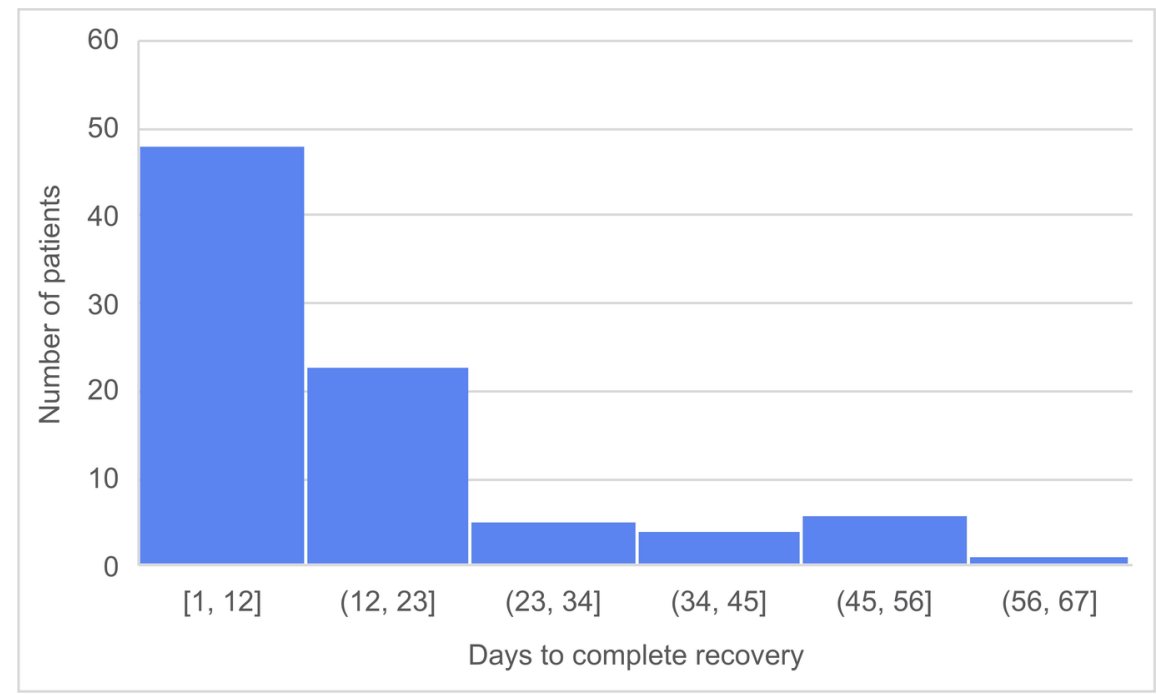

\section{Hosted file}

Table 1 Co.docx available at https://authorea.com/users/328896/articles/456026-prevalencerecovery-and-factors-associated-with-smell-and-taste-disruption-in-patients-withcoronavirus-disease-2019

\section{Hosted file}

Table 2 Co.docx available at https://authorea.com/users/328896/articles/456026-prevalencerecovery-and-factors-associated-with-smell-and-taste-disruption-in-patients-withcoronavirus-disease-2019

\section{Hosted file}

Table 3 CO.docx available at https://authorea.com/users/328896/articles/456026-prevalencerecovery-and-factors-associated-with-smell-and-taste-disruption-in-patients-withcoronavirus-disease-2019 